\title{
Dynamic 3D imaging of gas hydrate kinetics using synchrotron computed tomography
}

\author{
Zaher Jarrar ${ }^{1}$, Riyadh Al-Raoush ${ }^{2}$, Khalid Alshibli ${ }^{*}$, and Jongwon Jung ${ }^{3}$ \\ ${ }^{1}$ Department of Civil and Environmental Engineering, 325 John Tickle Building, University of Tennessee, Knoxville, TN, 37996 , USA. \\ ${ }^{2}$ Department of Civil and Architectural Engineering, Qatar University, P.O Box 2713, Doha, Qatar. \\ ${ }^{3}$ School of Civil Engineering, Chungbuk National University, Cheongju-si, Chungbuk 28644, Korea.
}

\begin{abstract}
The availability of natural gas hydrates and the continuing increase in energy demand, motivated researchers to consider gas hydrates as a future source of energy. Fundamental understanding of hydrate dissociation kinetics is essential to improve techniques of gas production from natural hydrates reservoirs. During hydrate dissociation, bonds between water (host molecules) and gas (guest molecules) break and free gas is released. This paper investigates the evolution of hydrate surface area, pore habit, and tortuosity using in-situ imaging of Xenon (Xe) hydrate formation and dissociation in porous media with dynamic three-dimensional synchrotron microcomputed tomography (SMT). Xe hydrate was formed inside a highpressure, low-temperature cell and then dissociated by thermal stimulation. During formation and dissociation, full 3D SMT scans were acquired continuously and reconstructed into 3D volume images. Each scan took only 45 seconds to complete, and a total of 60 scans were acquired. Hydrate volume and surface area evolution were directly measured from the SMT scans. At low hydrate saturation, the predominant pore habit was surface coating, while the predominant pore habit at high hydrate saturation was pore filling. A second-degree polynomial can be used to predict variation of tortuosity with hydrate saturation with an $\mathrm{R}^{2}$ value of 0.997 .
\end{abstract}

\section{Introduction}

Natural gas hydrates are ice-like structures in which a large amount of gas (mainly methane) is trapped within a crystal structure of water $[1,2]$. The availability of gas hydrates and the continuing increase in energy demand, motivated researchers to consider hydrates as a potential future source of energy. Gas production from natural hydrate reservoirs requires hydrate dissociation where bonds between water (host molecules) and gas (guest molecules) break and free gas is released. Hydrate dissociation can be affected by heat and mass transfer properties of porous media which can vary depending on permeability, relative permeability, and tortuosity [3-5]. In many classical hydrate dissociation models, hydrates are assumed to consist of a constant number of equalsized spheres dissociating at a constant rate [6,7]. Extensive research has been published on the use of computed tomography (CT) and SMT to characterize geomaterials. There is extensive literature about threephase (gas-fluid-solid) interaction with porous media using monochromatic SMT (e.g. [8-10]) where in situ SMT scans were acquired at different stages of the experiments. It is expensive to retrieve samples from natural marine environment while maintaining the high pressure and low temperature for an extended period of time and to image specimens using high resolution SMT since there is no enough $\mathrm{x}$-ray energy to penetration the sample container. As a result, many researchers studied laboratory-prepared hydrates using different gases. Industrial microfocus CT scanners have been used to study properties of laboratory-prepared hydrates (e.g. $[11,12])$ where it is challenging to separate different phases and monitor gas-solid-liquid contact properties since industrial CT scanners images do not have enough resolution.

This paper investigates the evolution of hydrate surface area, pore habit, and tortuosity using in-situ imaging of Xenon (Xe) hydrate formation and dissociation in porous media using dynamic 3D SMT. Xe hydrate was formed inside a high-pressure, lowtemperature cell and then dissociated by thermal stimulation. During dissociation, SMT scans were acquired continuously and reconstructed into $3 \mathrm{D}$ volume images.

\section{3D synchrotron micro-computed tomography (SMT)}

The use of a monochromator in SMT reduces the photon flux, which results in an increase in data collection time [13]. Alternatively, the beam can be reflected from a grazing incidence mirror which reflects energies below some cutoff that depends on the mirror coating and grazing incidence angle. This is referred to as "pink beam" and can result in a tunable broad bandwidth with

\footnotetext{
* Corresponding author: alshibli@,utk.edu
} 
more than 1000 times greater $x$-ray flux than a monochromatic beam [13]. The availability of highresolution cameras with fast-frame rate enabled the development of dynamic SMT using pink beam (PSMT) at beamline 13D, the Advanced Photon Source (APS), Argonne National Laboratory (ANL), Illinois. A pink beam can use up to $1 \mathrm{~ms}$ exposure time, which makes it ideal for monitoring dynamic processes such as hydrate dissociation $[13,14]$.

\section{Materials and experimental setup}

ASTM 20-30 Ottawa sand was used in this study. It is a natural silica sand with a grain size between US sieve \#20 $(0.841 \mathrm{~mm})$ and sieve \#30 $(0.595 \mathrm{~mm})$ and specific gravity of solids of 2.65 , mined and marketed by US Silica Company. The cell chamber is made from peek, a stong material with excellent x-ray attenuation, and has a height of $70.2 \mathrm{~mm}$, inner diameter of $9.7 \mathrm{~mm}$ (specimen diameter), wall thickness of $7.9 \mathrm{~mm}$ and two flanges. The cell can withstand an ultimate internal pressure up to $150 \mathrm{MPa}$ according to calculations based on peek manufacturer datasheet. A circulated refrigerant gas in the cooling coil near the end plate is used to cool the cell. Initially, the flow cell was partially filled with $4 \%$ by weight potassium iodide (KI) brine, and an equal amount of sand was deposited in four layers to achieve a target porosity of 0.40 . A steel mesh with $0.420 \mathrm{~mm}$ opening size was installed on the top of the specimen. Then, a plastic spacer and a stainless-steel spring were placed on the top of the steel mesh to provide a surcharge stress for the specimen. The flow cell was assembled and mounted on the stage of beamline 13D, APS (Fig. 1). More information about the experimental setup can be referred to in [15].

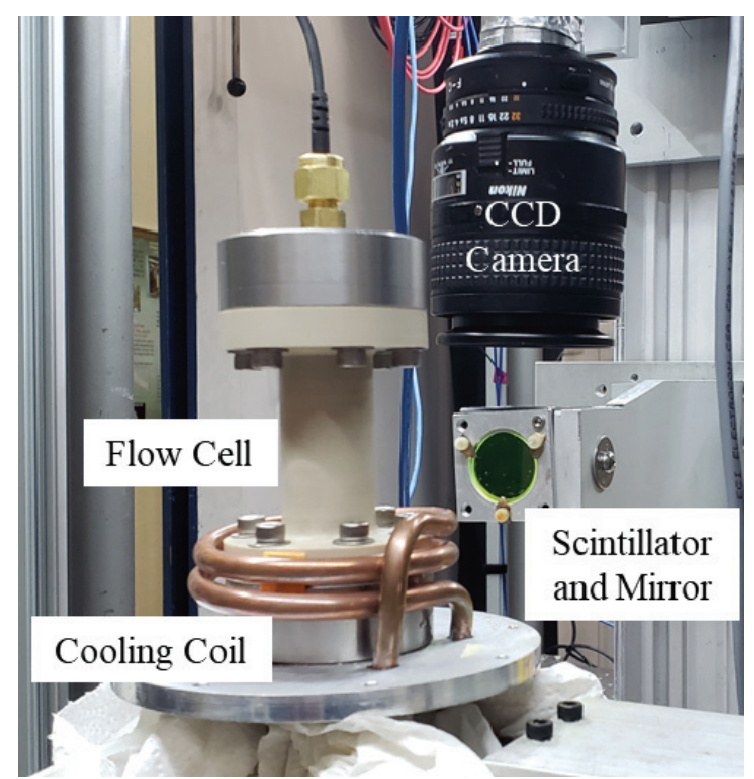

Fig. 1. Experimental setup at Beamline 13D, the Advanced Photon Source, Argonne National Laboratory.

\section{Results}

\subsection{Image reconstruction and analysis}

Image pre-processing and reconstruction were performed using the multi-threading software packcage "tomoRecon" [16]. Acquired images were processed using Avizo software [17]. The images have a spatial resolution of $5.38 \mu \mathrm{m} /$ pixel. Fig. 2 shows a sample 2D PSMT slice after 25 minutes of hydrate formation. First, a combination of anisotropic diffusion and median filters were used to reduce noise and enhance contrast of the images. The median filter reduce the contrast and soften the edges of the grayscale images by setting the pixel value to the median of the neighboring pixels [17]. Then, user-defined values of image intensity range were input to the interactive thresholding module in Avizo to isolate the hydrate phase pixels.

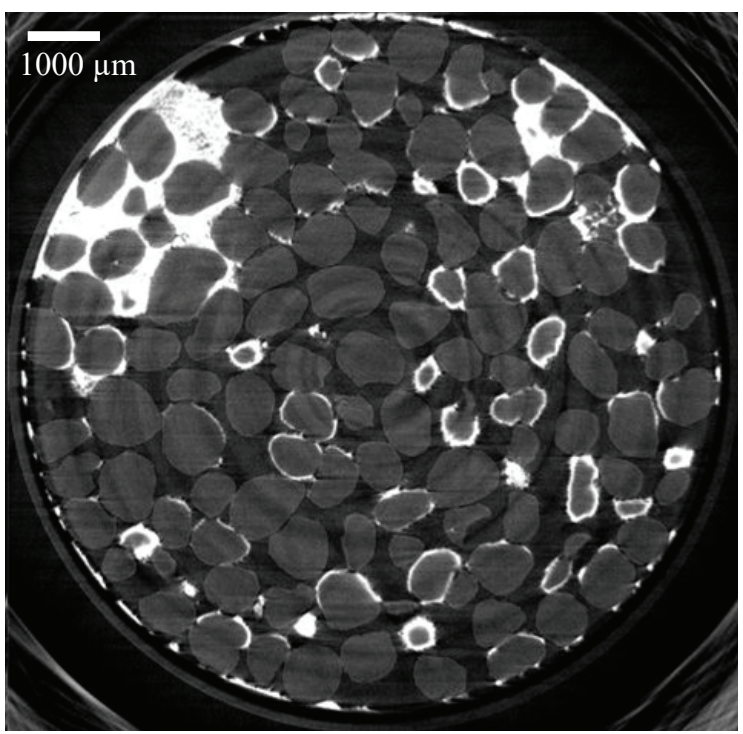

Fig. 2. Sample 2D PSMT slice after 25 minutes of hydrate formation.

\subsection{Surface area measurements during hydrate formation}

During the test, the temperature was controlled at $10^{\circ} \mathrm{C}$ $\pm 1^{\circ} \mathrm{C}$, and the initial Xe gas pressure was $1.9 \mathrm{MPa}$. Hydrate formation began instantly after the injection of Xe gas. PSMT imaging offers the advantage of directly quantifying hydrate volume and surface area during the test in a time resolution of seconds. Fig. 3 depicts the variation of hydrate specific area with hydrate saturation during Xe hydrate formation. Initially, specific area of hydrate increased with increasing hydrate saturation up to a threshold ( $10 \%$ hydrate saturation), after which specific area decreased with increasing hydrate saturation. Fig. 4 and Fig. 5 depict volume rendering snapshots of a vertical section of the specimen hydrate saturation of $7 \%$ and $24 \%$, respectively. The predominant hydrate pore-habit is surface coating for 
low hydrate saturation (Fig. 4). As hydrate saturation increases, hydrates start to change their pore-habit to pore-filling growing freely in the pore space (Fig. 5).

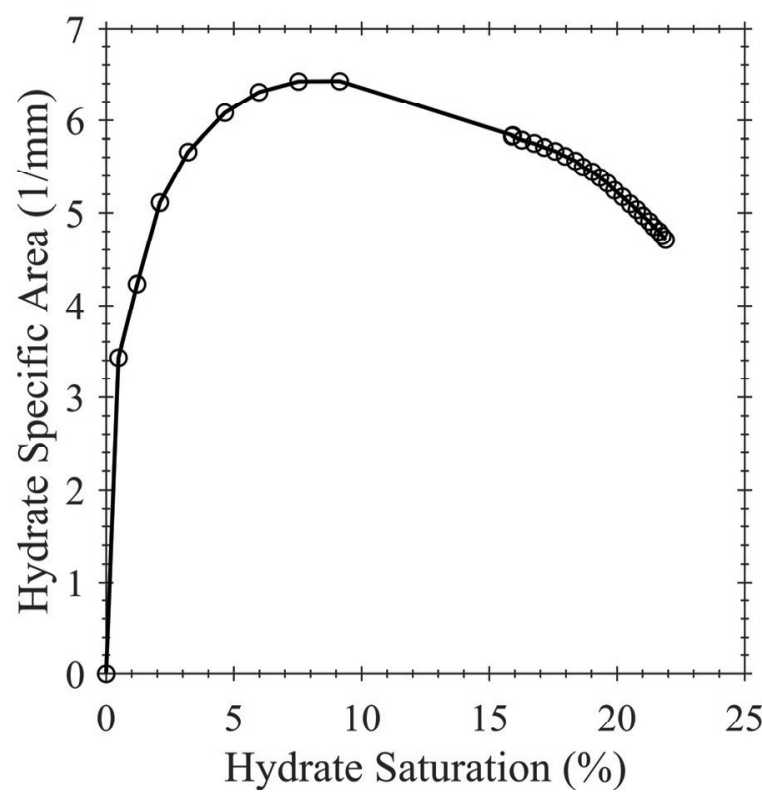

Fig. 3. Hydrate specific area changes with hydrate saturation during hydrate formation.

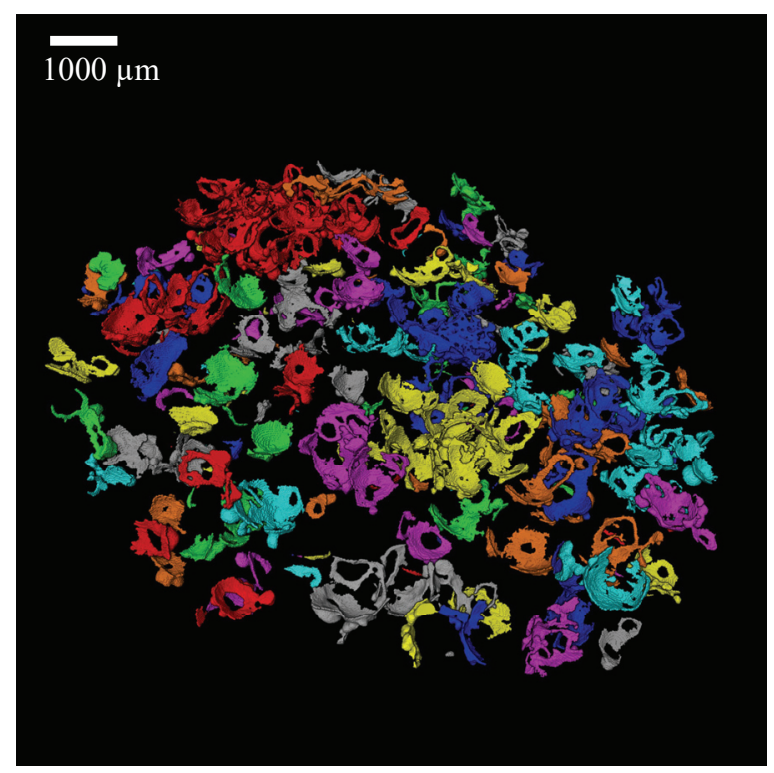

Fig. 4. Volume rendering snapshots at hydrate saturation of 7\% showing surface coating pore-habit.

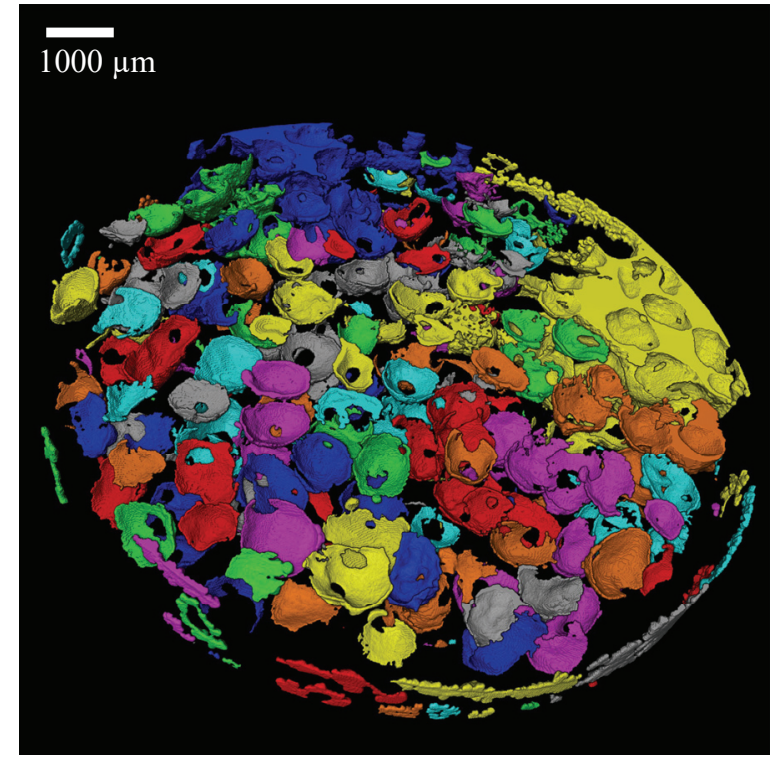

Fig. 5. Volume rendering snapshots at hydrate saturation of $24 \%$ showing pore filling pore-habit.

\subsection{Tortuosity evolution during hydrate dissociation}

Hydrate dissociation was triggered through thermal stimulation. Continuous full 3D PSMT was acquired during hydrate dissociation. Fig. 6 depicts the change in hydrate dissociation rate with hydrate saturation during the dissociation. Hydrate dissociation rate was measured experimentally by dividing the change in measured hydrate volume by the scan time between consecutive PSMT images. Tortuosity refers to the ratio of the average length of the geometrical paths through the medium and the straight-line length across the medium or the sample size. The tortuosity is due to many factors: the irregular morphology of the pore space, pores of different sizes and shapes, the local connectivity of the pores, and their complex spatial organization. Fig. 7 presents the variation of tortuosity with hydrate saturation during hydrate dissociation. During hydrate dissociation, measured tortuosity decreased with decreasing hydrate saturation (Fig. 7). A second-degree polynomial can be used to predict tortuosity with hydrate saturation with an $\mathrm{R}^{2}$ value of 0.997 .

\section{Conclusions}

This paper investigated the evolution of hydrate pore habit, surface area, and tortuosity during Xe hydrate formation and dissociation in porous media. Xe hydrate was formed inside a special flow cell that is capable of sustaining high pressure and low temperature. PSMT is a powerful tool that can be used to monitor dynamic processes such as hydrate formation and dissociation. Initially, specific area of hydrate increased with increasing hydrate saturation up to a threshold of $10 \%$ 


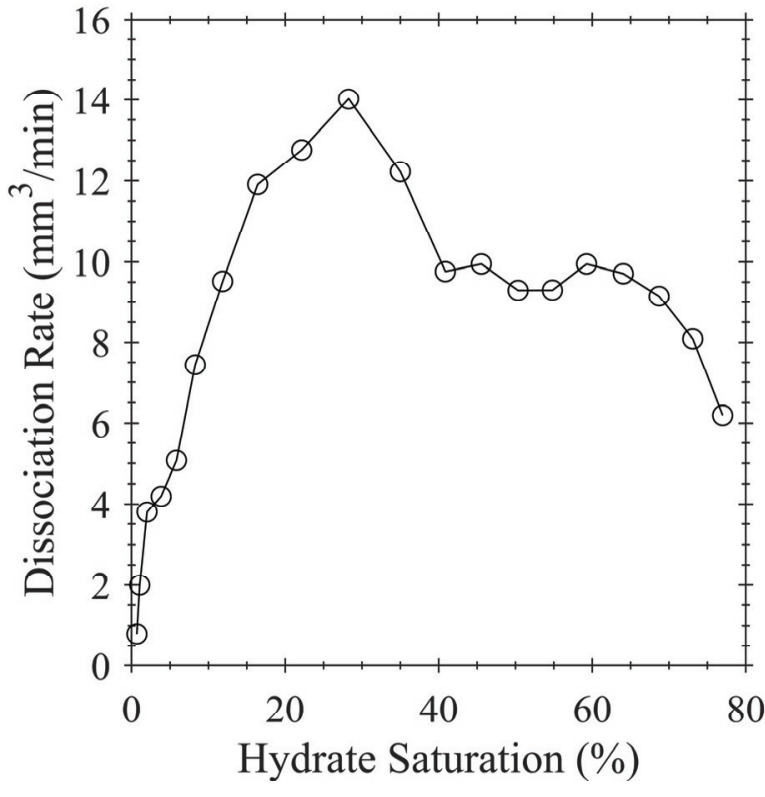

Fig. 6. Change of measured dissociation rate with hydrate saturation during hydrate dissociation.

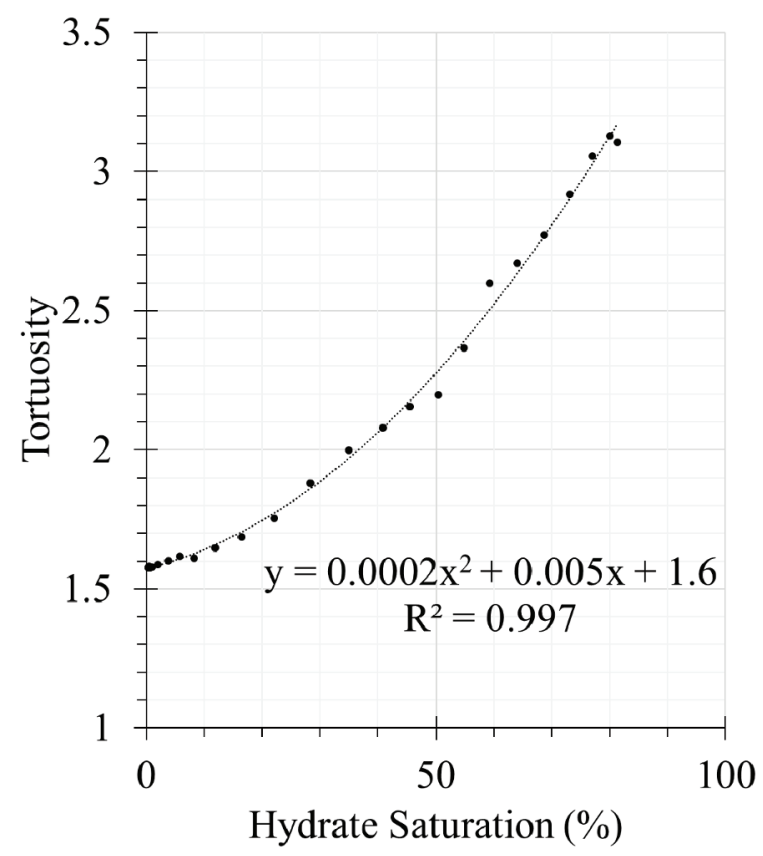

Fig. 7. Variation of tortuosity with hydrate saturation during hydrate dissociation.

saturation. After that, specific area decreased with increasing hydrate saturation. The predominant hydrate pore-habit was surface coating for low hydrate saturation, and pore-filling for high hydrate saturation. Hydrate dissociation rate was measured experimentally by dividing the change in measured hydrate volume by the scan time between consecutive PSMT images. The change of tortuosity with hydrate dissociation can be predicted using a second-degree polynomial with an $\mathrm{R}^{2}$ value of 0.997

This publication was made possible by partial funding from NPRP grant \# NPRP8-594-2-244 from the Qatar national research fund (a member of Qatar Foundation) and the Institute for a Secure and Sustainable Environment (ISSE), University of Tennessee-Knoxville, USA. Any opinions, findings, and conclusions or recommendations expressed in this material are those of the authors and do not necessarily reflect the views of funding agencies. The authors would like to thank Mr. Wadi Imseeh for his help during scanning and Mr. Jamal Hannun for his help in interfacial area analysis. This paper used resources of the Advanced Photon Source (APS), a U.S. Department of Energy (DOE) Office of Science User Facility operated for the DOE Office of Science by Argonne National Laboratory (ANL) under Contract No. DE-AC02-06CH11357. The PSMT images presented in this paper were collected using the $\mathrm{x}$-ray Operations and Research Beamline Station 13-BMD at Argonne Photon Source (APS), ANL. We thank Dr. Mark Rivers of APS for help in performing the PSMT scans. We also acknowledge the support of GeoSoilEnviroCARS (Sector 13), which is supported by the National Science Foundation, Earth Sciences (EAR-1128799), and the DOE, Geosciences (DEFG02-94ER14466).

\section{References}

1. E. Sloan, C. Koh, Clathrate hydrates of natural gases CRC press (2007)

2. M. Max, A. Johnson, W. Dillon, Natural gas hydrate-arctic ocean deepwater resource potential Springer (2013)

3. V. Prasad and F. Kulacki, Convective heat transfer in a rectangular porous cavity-effect of aspect ratio on flow structure and heat transfer. J. Heat Tranf. 106 158-165 (1984)

4. O. Trevisan and A. Bejan, Natural convection with combined heat and mass transfer buoyancy effects in a porous medium. Int. J. Heat Mass Transf. 28 1597-1611 (1985)

5. M. Kaviany, Principles of heat transfer in porous media. Springer Science \& Business Media (2012)

6. H. Kim, P. Bishnoi, R. Heidemann, S. Rizvi, Kinetics of methane hydrate decomposition. Chem. Eng. Sci. 42 1645-1653 (1987)

7. X. Chen and D. Espinoza, Surface area controls gas hydrate dissociation kinetics in porous media. Fuel 234 358-363 (2018)

8. M. Thakur, D. Penumadu, C. Bauer, Capillary suction measurements in granular materials and direct numerical simulations using x-ray computed tomography microstructure. J. Geotech. Geoenviron. 14604019121 (2020)

9. Z. Jarrar, R. Al-Raoush, J. Hannun, K. Alshibli, J. Jung, 3D synchrotron computed tomography study on the influence of fines on gas driven fractures in sandy sediments. Geom. Ener. Env. 100105 (2018)

10. A. Herring, L. Andersson, D. Wildenschild, Enhancing residual trapping of supercritical $\mathrm{CO} 2$ via 
cyclic injections. Geophys. Res. Lett., 43 9677-9685 (2016)

11. M. Sato, S. Takeya, J. Nagao, S. Jin, Y. Kamata, H. Minagawa, T. Ebinuma, H. Narita, Distribution of hydrate saturation ratios in artificial methane hydrate sediments measured by high-speed X-ray computerized tomography. Jpn. J. Appl. Phys. 44 473 (2005)

12. L. Lei, Y. Seol, J. Choi, T. Kneafsey, Pore habit of methane hydrate and its evolution in sediment matrix-laboratory visualization with phase-contrast micro-CT. Mar. Petrol. Geol. 104 451-467 (2019)

13. M. Rivers, Developments in X-Ray Tomography X, SPIE (2016)

14. Z. Jarrar, K. Alshibli, R. Al-Raoush, J. Jung, 3D measurements of hydrate surface area during hydrate dissociation in porous media using dynamic 3D imaging. Fuel 265, 116978 (2020)

15. K. Alshibli and Z. Jarrar, Four-dimensional dynamic synchrotron microcomputed tomography imaging of gas-water interface at high pressure and low temperature. Geotechnical Testing Journal 44-4, in press (2021)

16. M. Rivers, Developments in $x$-Ray tomography VIII International society for optics and photonics (2012)

17. Avizo 9.7. FEI, Hillsboro, OR. 